\title{
Hidden Markov Model for Exchange Rate with EWMA Control Chart
}

\author{
$1^{\text {st }}$ Rahmawati Ramadhan ${ }^{1}, 2^{\text {nd }}$ Dodi Devianto ${ }^{2}, 3^{\text {nd }}$ Maiyastri $^{3}$ \\ \{rahmawatiramadhan70@gmail.com ${ }^{1}, \underline{\text { ddevianto@ @si.unand.ac.id }}$, maiyastri@ gmail.com ${ }^{3}$ \}
}

Department of Mathematics, Andalas University, Padang, 25163, Indonesia ${ }^{1,2,3}$

\begin{abstract}
Nowadays, the US dollar exchange rate is still very influential on the exchange rate stability of many countries, including Indonesia. The effect of the US Dollar exchange rate has caused the fluctuation of Rupiah exchange rate. That is one of the cases that can be modeled with the Hidden Markov Model (HMM) as the development of a Markov chain in which its state is not able to be observed directly (hidden), but it is only able to be observed through a set of other observations. In this paper, Exponentially Weighted Moving Average (EWMA) control chart will be used to determine the state of HMM. Based on the EWMA control chart, there are three states which are increase, decrease, and constant. The probability of the changes of exchange rate will be predicted in 2019 with the Baum Welch Algorithm on HMM. By using 240 exchange rate data of US Dollar to Rupiah in 2018, it is predicted the changes of exchange rate in 2019 are increased with a probability of 0.57. The results of HMM have connected to the EWMA control chart where they have eight uncontrolled data with two states increase and six states decrease. Thus, the existence of uncontrolled data implies the probability of increasing of the exchange rate in 2019.
\end{abstract}

Keywords: exchange rate, exponentially weighted moving average, hidden Markov model

\section{Introduction}

Exchange rates define the rate or ratio of which one of these currencies can be exchanged for any other at any given point in time. The fluctuation of the exchange rate is always influenced by many factors, such as the diversity of the inflation and the interest rate between two countries, the trade balance,public debt, the ratio of export prices and import prices, with political and economic stability [1].

In analyzing the fluctuations of the future exchange rate, it can be attributed to the stochastic problem, where the problem is related to the probability of occurrence in the future cannot be predicted certainly. Because it forms Markov chain, then Markov chain is supposedly unobserved such that the problem of exchange rate fluctuations can be modeled by Hidden Markov Models.

Hidden Markov Model (HMM) is a Markov chain that the sequence of states is unobserved. In Hidden Markov Models, there are three fundamental problems to be solved that are evaluations problem, decoding problems, and learning problems. Based on previous research, number of creatures of a species found within a river using negative binomial hidden Markov models as discussed in [2]. According to research in [3], [4], and [5], hidden Markov model is also related to Bayesian Analysis. In time series cases, there are many researches 
which studied about hidden Markov model. One of them discussed robust classification of multivariate time series as explained in [6], categorical time series which introduced in [7], and time series modeling for risk also obtained in [8]. Moreover, to predict a trend in financial time series using hidden Markov model was also as discussed in [9]. Based on the research in [10], the technique to build a model by using financial data can be used Autoregressive Fractionally Integrated Moving Average (ARFIMA). Furthermore, by using financial data will be determined the model of the most efficient optimal portfolio selection also explained in [11], and cluster analysis was proved to see a homogeneous of the financial performance which is described in [12].

Therefore, this research will present Graphics Controller Exponentially Weighted Moving Average (EWMA) that determines the state of exchange rate which the data are used to predict the probability of increasing the exchange rate for the period of the next day using a Hidden Markov Model with evaluation, decoding and learning problem. EWMA control chart has related to exponential distribution where the characterization of exponential distribution is explained in term of convolution and characteristic function such as described in [13], [14], [15], and [16]. The result gives simplicity to take decisions in investment.

\section{Graphics Controller Exponentially Weighted Moving Average (EWMA) with Hidden Markov Model}

Exponentially Weighted Moving Average (EWMA) control chart is one of the statistical quality control techniques are used to monitor the quality of the products of a production process. In this case, the EWMA control chart will be used to detect the state of a data exchange rate in 2018 that taken from the difference between the current exchange rate value and the previous one. The following EWMA control charts exchange rate.

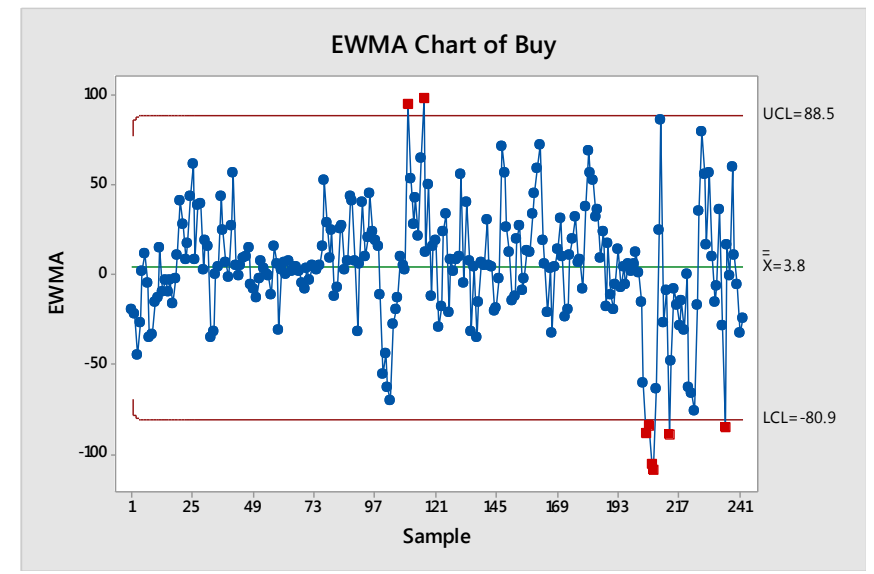

Fig. 1.EWMA Control Charts Exchange Rate

Consider on Fig. 1, it shows that there is a transition state of the current exchange rate value, namely increase, decrease, and constant. This state will be set as a condition to identify the exchange rate value in 2019 by using the Hidden Markov Model. The set of ordered data 
with number $110,116,204,205,206,207,213$, and 235 indicate that the data are not well controlled. On the data to 110 , its state is increasing, meaning that the value of the exchange rate at the previous data is lower than the value of the exchange rate at the data afterwards. While the state on the data to 116 and 204 are increasing, and to 205, 206, 207, 213, and 235 are decrease. Thus, there are uncontrolled data then it will affect the probability for increasing the exchange rate in 2019.

\section{Hidden Markov Model (HMM)}

The stochastic process is a sequence of events that qualifiers the laws of probability where every value changed againts time in random manner. A stochastic process which predicted future traits depending on the properties of a present-day variable based on its characteristics in the past is called the Markov chain [17]. A stochastic process $X_{n}$ has Markov properties, if

$$
P\left(X_{n+1}=j \mid X_{n}=i, X_{n-1}=i_{n-1}, \ldots, X_{0}=i_{0}\right)=P\left(X_{n+1}=j \mid X_{n}=i\right)
$$

for a time $n=0,1, \ldots$ and for every $j, i, i_{n-1}, \ldots, i_{1} i_{0}$.

Hidden Markov Model (HMM) is a stochastic model where the system is assumed to be a Markov Process with hidden states.If $X=\left\{X_{1}, X_{2}, \ldots\right\}$ is a Markov process, and $O=\left\{O_{1}, O_{2}, \ldots\right\}$ is a function of $X, X$ it is a Hidden Markov Model which can be observed through $O$, or can be written to a function $f$. Parameter $X$ denotes a state process that is hidden, while parameter $O$ denotes an observation space that can be observed. The elements of the Hidden Markov Model are:

1. The number of hidden state elements (hidden state) represented by $N$ as the number of states which the probability of a denoted state space $S=\left\{S_{1}, S_{2}, \ldots, S_{N}\right\}$ and the state at the time tdenoted by $X_{t}, t=1,2, \ldots, T$

2. The number of observations (observation) of each state represented by $M$, where probability every state represented by $v=\left\{v_{1}, v_{2}, \ldots, v_{M}\right\}$ and space observation represented by $O=\left\{O_{1}, O_{2}, \ldots, O_{T}\right\}$, which $T$ is the length of the observation data.

3. Transition probability matrix, $A=\left\lfloor a_{i j}\right\rfloor$ Where $a_{i j}$ is element of $A$ which is the conditional probability of the state at the time $t+1$, given the stateXat the time $\mathrm{t}$, that is

$$
a_{i j}=P\left(X_{t+1}=j \mid X_{t}=i\right)
$$

for $1 \leq i, j \leq N$

4. Observation probability distribution at the time $t$, at state $j$, commonly known as emission matrix

$$
B=\left[b_{i k}\right]
$$

where

$$
b_{i k}=P\left(O_{t}=v_{k} \mid X_{t}=i\right),
$$

for $1 \leq j \leq N, 1 \leq t \leq T$ and $1 \leq k \leq M$

5. The initial state distribution represented by $\pi(i)$

where:

$$
\pi(i)=P\left(X_{1}=i\right), 1 \leq i \leq N
$$


Thus, HMM can be written in the form $\lambda=(A, B, \pi)$ which is $A$ denotes transition probability matrix, $B$ denotes matrix of observation probability and also known as emission matrix, and $\pi$ is the distribution of the initial state. There are three special problems that can be solved by Hidden Markov Model methods, then

a) Evaluation Problem

To calculate the probability of the observation sequence $P(O \backslash \lambda)$ needed the forward algorithms and the backward algorithm [17]. This algorithm is an iterative process that is based on the calculation of conditional probability through properties on probability [18].

First, calculate the probability of observation using the forward algorithm $\alpha_{t}(i)$. Generally it consists of the following 3 steps:

i. Initialization

In this initialization stage, the initial observation probability $\alpha_{1}(i)$ will be determined, as follows

for $1 \leq i \leq N$

$$
\alpha_{1}(i)=\pi(i) b_{i}\left(O_{1}\right)
$$

ii. Induction

In this stage, the total probabilities for observation at $t>1$ will be calculated as follow

$$
\alpha_{t+1}(j)=\left\{\sum_{i=1}^{N} \alpha_{t}(i) a_{i j}\right\} b_{j}\left(O_{t+1}\right)
$$

for $j=1,2, \ldots, N, t=1,2, \ldots, T$

iii. Terminations

This step is to sum all the probabilities for the observation sequence as follow

$$
P(O \mid \lambda)=\sum_{i=1}^{N} \alpha_{T}(i)
$$

Second, calculate the probability of observation using the backward algorithm $\beta_{t}(i)$. Generally it consists of the following 3 steps:

i. Initialization

At this stage, the probability for observation is stated as equal to one, because it is assumed $i$ to be the final state and is zero for $i$ the others as follows

$$
\beta_{t}(i)=1
$$

for $1 \leq i \leq N$

ii. Induction

In this stage, the total probabilities for observation will be calculated at $t<1$ as follows

$$
\beta_{t}(i)=\sum_{j=1}^{N} a_{i j} b_{j}\left(O_{t+1}\right) \beta_{t+1}(j)
$$

for $t=T-1, T-2, \ldots, 1$ and $i=1,2, \ldots, N$

iii. Terminations

This step is to sum all the probabilities for the observation sequence as follows

$$
P(O \mid \lambda)=\sum_{i=1}^{N} b_{i}(1) \pi(1) \beta_{1}(i)
$$

b) Decoding Problem

Decoding problemis to find the best state sequence (optimal) associated with the observation $O$ of the models $\lambda$ that are also known. The probability of the most optimal sequence for this observation sequence is denoted by $\delta_{t}(i)$. While $\varphi_{1}(j)$ saving the previous 
state which will form the most optimal state sequences. This problem can be solved by the Viterbi Algorithm which consists of four stages [17], as follows

i. Initialization

This initial stage will determine the sequence of hidden conditions at $t=1$, as follows

$$
\begin{aligned}
& \delta_{1}(i)=b_{i}\left(O_{1}\right) \pi(i) \\
& \varphi_{1}(i)=0, \text { for } 1 \leq i \leq N
\end{aligned}
$$

ii. Recursion

The next stage is to determine the sequence of the hidden conditions at $t \geq 2$, as follows

$$
\delta_{t}(j)=\max _{1 \leq i \leq N}\left\{\delta_{t-1}(i) a_{i j}\right\} b_{j}\left(O_{t}\right)
$$

for $2 \leq t \leq T$, and $1 \leq j \leq N$

$$
\varphi_{1}(j)=\underset{1 \leq i \leq N}{\arg \max }\left\{\delta_{t-1}(i) a_{i j}\right\}
$$

for $2 \leq t \leq T$, and $1 \leq j \leq N$

iii. Terminations

In this stage, the greatest probabilities along $t$ will be calculated for the last observation, as follows

$$
\begin{aligned}
& P^{*}=\underset{1 \leq i \leq N}{\max }\left\{\delta_{T}(i)\right\} \\
& X_{T}^{*}=\underset{1 \leq i \leq N}{\arg \max }\left\{\delta_{T}(i)\right\}
\end{aligned}
$$

iv. Backtracking

In the last stages, the best state sequences will be determined, as follows

$$
X_{t}^{*}=\varphi_{t+1}\left(X_{t+1}^{*}\right), t=T-1, T-2, \ldots, 1
$$

c) Learning problems

This problem is estimating the best model that can represents a sequence of observations which is the changing parameters of $\operatorname{HMM}, \pi=(A, B, \pi)$ that $P(O \mid \lambda)$ to be maximum.

In the Baum-Welch algorithm, also defined four variables, that are: Forward variable, Backward variable, variable $\xi_{t}(i, j)$, and variable $\gamma_{t}(i)$.Forward and backward variables will be used in the calculation of the variable $\xi_{t}(i, j)$ and variable $\gamma_{t}(i)$. The Variable $\xi_{t}(i, j)$ is the probability of the process in the state $i$ at the time $t$ and in the state $j$ at the time $t+1$. While the variable $\gamma_{t}(i)$ is the total of $\xi_{t}(i, j)$.So that the estimation formula learning problem as follows:

$$
\begin{aligned}
& \hat{\pi}(i)=\gamma_{t}(i), 1 \leq i \leq N \\
& \hat{a}_{i j}=\frac{\sum_{t=i}^{T-1} \xi_{t}(i, j)}{\sum_{t=1}^{T-1} \gamma_{t}(i)}, 1 \leq i \leq N, 1 \leq j \leq N
\end{aligned}
$$




\section{Methods}

\subsection{Data}

In this research, the exchange rate US Dollar to Rupiah data used only one year that is in 2018, from January 1, 2018 to December 31, 2018. The data are provided at http://bi.go.id with number of data is 240 .

\subsection{Analysis methods}

This research uses Hidden Markov Model. That will be analyzed is exchange rate data with predictions of probability exchange rate on the next year, as follows:

1. Take exchange ratedata by a period of one day. The number of data must be 240 data.

2. Determine transition probability matrix neededexchange rate by using the following formula as a probability.

$$
P(A)=\frac{n(A)}{n(S)}
$$

where $n(A)$ is the number of elements in $A$ and $n(S)$ is the total number of elements in $S$ [18].

3. Determine elements of Hidden Markov Model.

4. Analyze the elements of Hidden Markov Models that have been able to use three problems in the Hidden Markov Model is calculate the probability of an observation by using Forward-Backward algorithm, determines the sequences state of hidden by using the Viterbi algorithm, and predicting the HMM parameters using the Baum-Welch algorithm.

5. Make the interpretations or conclusions from results that have been obtained.

\section{Results and Discussion}

Hidden Markov Models (HMM) are based on the well-known theories of Markov chains, where the states are assumed hidden. HMM is also been utilized on financial data such as exchange rate. To predict the probability of the changes of exchange rate in 2019 by using HMM as following steps:

1. The elements of the Hidden Markov Model

a. Suppose $N$, denoted by the number of hidden state, with state space $S=\left\{S_{1}, S_{2}, \ldots, S_{N}\right\}$ and the state at the time $t$ denoted by $X_{t}$. In this case of exchange rate, hidden state is increase, decrease, and constant. So in this case $N=3$ or be written by $S_{1}=P$ (increase), $S_{2}=P$ (decrease), $S_{3}=P$ (constant). For example, $X_{t}=1$ that is states which are in an increasing state.

b. Suppose $M$, that is the number of observations of each state, the observation space $O=\left(O_{1}, O_{2}, \ldots, O_{T}\right)$ and probability of each state observation represents by $v=\left\{v_{1}, v_{2}, \ldots v_{M}\right\}$, in this research $M=1$ exchange rate as $\left(v_{1}\right)$.

c. Suppose $A=a_{i j}=P\left(X_{t+1}=j \mid X_{t}=i\right)$ is probability of the exchange rate in the range to $i$ in week $t+1$ if it is known in the week to be in the range of values to that probability matrix formed by: 


$$
A=\left[a_{i j}\right]=\left[\begin{array}{lll}
a_{11} & a_{12} & a_{13} \\
a_{21} & a_{22} & a_{23} \\
a_{31} & a_{32} & a_{33}
\end{array}\right]
$$

Transition Probability Matrix exchange rate data

$$
A=\left[a_{i j}\right]=\left[\begin{array}{ccc}
0.52 & 0.44 & 0.04 \\
0.51 & 0.49 & 0 \\
0.5 & 0.33 & 0.17
\end{array}\right]
$$

d. $B=\left[b_{i k}\right]$ is conditional observation probability matrix $v_{k}$ if the process at state $j$, the observation matrix for the exchange rate is asfollows:

$$
B=\left[b_{i k}\right]=\left[\begin{array}{c}
0.52 \\
0.46 \\
0.02
\end{array}\right]
$$

e. Suppose $\pi(i)$ is the initial state distribution, in case of the exchange rate is assumed by:

$$
\pi(1)=P(\text { increase }), \pi(2)=P(\text { decrease }), \pi(3)=P(\text { constant })
$$

Initial matrix for the exchange rate is as follows:

$$
\pi=\left[\begin{array}{l}
0.33 \\
0.33 \\
0.34
\end{array}\right]
$$

2. Evaluation Problem Forward and Backward Algorithm

For the first problem of the Hidden Markov Model, it will be calculated the probability model $\lambda=(A, B, \pi)$ that represented by $P(O \mid \lambda)$ or the probability of the observation sequences $O=\left\{O_{1}=v_{1}\right\}$. This probability can be determined by using the Forward and Backward algorithms.

$$
\begin{aligned}
P(O \mid \lambda) & =\sum_{i=i}^{N} a_{T}(i) \\
& =a_{2}(1)+a_{2}(2)+a_{2}(3)=0.0884+0.0699+0.0002=0.1585 \\
P(O \mid \lambda) & =\sum_{i=1}^{N} \beta_{1}(i) \pi(i) b_{i}\left(O_{1}\right. \\
& =\beta_{1}(1) \pi(1) b_{1}\left(O_{1}\right)+\beta_{1}(2) \pi(2) b_{2}\left(O_{1}\right)+\beta_{1}(3) \pi(3) b_{3}\left(O_{1}\right) \\
& =(0.4736)(0.33)(0.52)+(0.4906)(0.33)(0.46)+(0.4152)(0.34)(0.02) \\
& =0.1585
\end{aligned}
$$

Based on the results of the backward algorithm, the probability of this algorithm is equal to the probability of the forward algorithm that is 0.1585 .

3. Decoding Problem with the Viterbi Algorithm

Decoding problem is how to determine the optimal hidden state sequence as follows increase, decrease, or constant of exchange rate, compatible with a sequence of observations that have been assumed. Viterbi algorithm consists of two steps, including the following: 


$$
\begin{aligned}
& X_{1}^{*}=1 \\
& X_{2}^{*}=1
\end{aligned}
$$

It means that the most suitable sequence of the increased, decreased, or constant exchange ratesequences in 2019 is the increase sequence.

4. Learning problems with Algorithm Baum Welch

To calculate the parameters of HMM prediction using Baum Welch algorithm can be defined a new variable $\xi_{t}(i, j)$, that probability process in state $j$ at the time $t+1$. It following

$$
\hat{\pi}=\left[\begin{array}{l}
\gamma_{1}(1) \\
\gamma_{1}(2) \\
\gamma_{1}(3)
\end{array}\right]=\left[\begin{array}{l}
0.5126 \\
0.4697 \\
0.0177
\end{array}\right]
$$

The value $\gamma_{t}(i)$ for $t=1$ is to estimate of the initial probability. That means the value $P(O \mid \hat{\lambda}) \geq P(O \mid \lambda)$ is complete, then theprobability process in state of exchange rate will increase by 0.5126 , probability of the exchange rate will decreaseby 0.4697 and the estimated initial probability processof exchange rate will constant by 0.0177 .

Meanwhile, a state prediction $\hat{a}_{i j}$ of the transition probability matrix $a_{i j}$ is a comparison between the number of transition probability from state $i$ to state $j$ with the displacement probability in the state $i$, its following:

$$
\begin{array}{r}
\hat{a}_{i j}=\left[\begin{array}{ccc}
\frac{\sum_{t=1}^{T} \xi_{t}(1,1)}{\sum_{t=1}^{T} \gamma_{t}(1)} & \frac{\sum_{t=1}^{T} \xi_{t}(1,2)}{\sum_{t=1}^{T} \gamma_{t}(1)} & \frac{\sum_{t=1}^{T} \xi_{t}(1,3)}{\sum_{t=1}^{T} \gamma_{t}(1)} \\
\frac{\sum_{t=1}^{T} \xi_{t}(2,1)}{\sum_{t=1}^{T} \gamma_{t}(2)} & \frac{\sum_{t=1}^{T} \xi_{t}(2,2)}{\sum_{t=1}^{T} \gamma_{t}(2)} & \frac{\sum_{t=1}^{T} \xi_{t}(2,3)}{\sum_{t=1}^{T} \gamma_{t}(2)} \\
\frac{\sum_{t=1}^{T} \xi_{t}(3,1)}{\sum_{t=1}^{T} \gamma_{t}(3)} & \frac{\sum_{t=1}^{T} \xi_{t}(3,2)}{\sum_{t=1}^{T} \gamma_{t}(3)} & \frac{\sum_{t=1}^{T} \xi_{t}(3,3)}{\sum_{t=1}^{T} \gamma_{t}(3)}
\end{array}\right] \\
=\left[\begin{array}{llll}
\frac{0,2927}{0,5126} & \frac{0,2191}{0,5126} & \frac{8,67.10^{-4}}{0,5126} \\
\frac{0,2539}{0,4697} & \frac{0,2158}{0,4697} & \frac{0}{0,4697} \\
\frac{0,0111}{0,0177} & \frac{6,51.10^{-3}}{0,0177} & \frac{1,46.10^{-4}}{0,0177}
\end{array}\right] \\
=\left[\begin{array}{ccc}
0,57 & 0,42 & 0,01 \\
0,54 & 0,46 & 0 \\
0,62 & 0,36 & 0,02
\end{array}\right]
\end{array}
$$

Matrix $\hat{a}_{i j}$ is an estimator for the transition matrix $a_{i j}$. The matrix $\hat{a}_{i j}$ showed that to achieve the value $P(O \mid \hat{\lambda}) \geq P(O \mid \lambda)$ then the transition probability of exchange rate are "increase" to "increase" is 0.57 , from "increase" to "decrease" at 0.42 , from "increase" to "constant" is 0.01 , and from "decrease" to "increase" at 0.54 , from "decrease" to "decrease" 0.46 , from "decrease" to "constant" is 0 , and from "constant" to "increase" by 0.62 , from "constant" to "decrease" at 0.36 , from "constant" to "constant" by 0.02 . 
Thus the probability of increase in exchange rate in 2019 is 0.57 . Whereas, the probability of decrease is 0.42 and constant is 0.01 . It means 2019 might be probability for the exchange rate will be more increase. It means that if the exchange rate become increasing so many aspects especially economic sectors will be changes.

\section{Conclusion}

Hidden Markov model with the Viterbi algorithm shows that 2019 might be probability for the exchange rate will be more increasing that are equal to 0.57. Relations with EWMA chart, the graph showsthe transition state ofexchange rate data in 2018 and also there are some data uncontrollably. If more data are uncontrolled,the probability ofthe increase exchange rate is become greater. It means that the increase exchange rate will give negative impact to economic sector in Indonesia, such as the import products become more expensive, spurring inflation growth, foreign investors suffered losses, and increasing debt burden of government.

\section{References}

[1] Wulan, E. R, and Nurfaiza, S.:Analysis of Factors Affecting Inflation in Indonesia: an Islamic Perspective. International Journal of Nusantara Islam.Vol 2(2), pp. 67-80 (2014)

[2] Spezia, L, S. L. Cooksley, M.J. Brewer, D. Donnelly, and A. Tree.: Modelling Species Abundance in a River by Negative Binomial Hidden Markov Models.Computational Statistics and Data Analysis. Vol. 71, pp. 599-614 (2014)

[3] Xia, Y. M, and Nian Sheng Tang.: Bayesian Analysis for Mixture of Latent Variabel Hidden Markov Models with Multivariate Longitudinal data.Computational Statistics and Data Analysis. Vol. 5, pp. 190-211 (2019)

[4] Li, X,ViliamMakis, HongfuZuo, and Jing Cai.: Optimal Bayesian Control Policy for Gear Shaft Fault Detection using Hidden Semi-Makov Model. Computational Statistics and Data Analysis. Vol. 119, pp. 21-35 (2018)

[5] Bathaee, $\mathrm{N}$ and HamisSheikhzadeh.: Non-Parametric Bayesian Inference for Continous Density Hidden Markov Mixture Model.Statistical Methodology. Vol. 33, pp. 256-275 (2016)

[6] Antonucci, A.: Robust Classification of Multivariate Time-Series by Imprecise Hidden Markov Model.International Journal of Aproximate Reasoning. Vol. 56, pp 249263 (2015)

[7] Columbi, R and S. Giordano.: Multiple Hidden Markov Models for Caterogical Time-series. Journal of Multivariate Analysis. Vol. 140, pp. 19-30 (2015)

[8] Devianto, D, Maiyastri, and DR. Fadhilla.: Time Series Modelling for Risk with Value at Risk Computation.Apllied Mathematical Sciences. Vol. 9(56), pp. 2779-2787 (2015)

[9] Zhang, M, Xin Jiang, Zehua Fang, YueZeng, and KeXu.: High-Order Hidden Markov Model for Trend Prediction in Financial Time Series.Physica A. Vol. 517, pp. 112 (2019)

[10] Devianto, D, Maiyastri, and S. Damayanti.: Forecasting Long Memori Time Series for Stock Price with Autoreggressive Fractionally Intergrated Moving Average.International Journal of Applied Mathematics and Statistics. Vol. 53(5), pp. 8695 (2015) 
[11] Devianto, D, Maiyastri, Randy, Masyhuri Hamidi, Sri Maryati, and Afridian Wirahadi Ahmad.:Efficiency Analysis of Optimal Portfolio Selection for Stocks in LQ45 Index. IEEE Explore. pp. 78-83(2019)

[12] Nazar, M. F,Maiyastri, Dodi Devianto, and Hazmira Yozza.: On the Clustering of Islamic Rural Banks Based on Financial Performance. IEEE Explore. pp. 108-113 (2019)

[13] Devianto, D.: On the Class of Infinitely Divisible Exponential Distribution. Journal of Physics: Conference Series 1097012084 (2018)

[14] Devianto, D.: The Uniform Continuity of Characteristic Function from Convoluted Exponential Distribution with Stabilizer Constant. AIP Conference Proceedings 1707, American Institute of Physics, New York. pp. 1-5 (2016)

[15] Devianto, D. ,Maiyastri, L. Oktasari and M. Anas.: Convolution of Generated Random Variable from Exponential Distribution with Stabilizer Constant. Applied Mathematical Sciences. Vol. 9 (96), pp. 4781-4789 (2015)

[16] Devianto, D., L. Oktasari and Maiyastri.: Some Properties of Hypoexponential Distribution with Stabilizer Constant. Applied Mathematical Science. Vol.9 (142), pp. 7063-7070 (2015)

[17] Ross, Sheldon M.:Stochastic Process. United States of America (1982)

[18] Bain, LJ and M. Engelhardt.: Introduction to Probability andMathematical Statistics. Second Edition, Duxbury Press, California (1992) 